Coupled Pressure-Temperature Probes for Monitoring Porewater Fluxes in Coastal Sediment Nicole K. LeRoux ${ }^{1,2}$, Joseph Tamborski ${ }^{1,3}$, Sanjana Moodbagili,4, \& Barret L. Kurylyk ${ }^{1,2}$

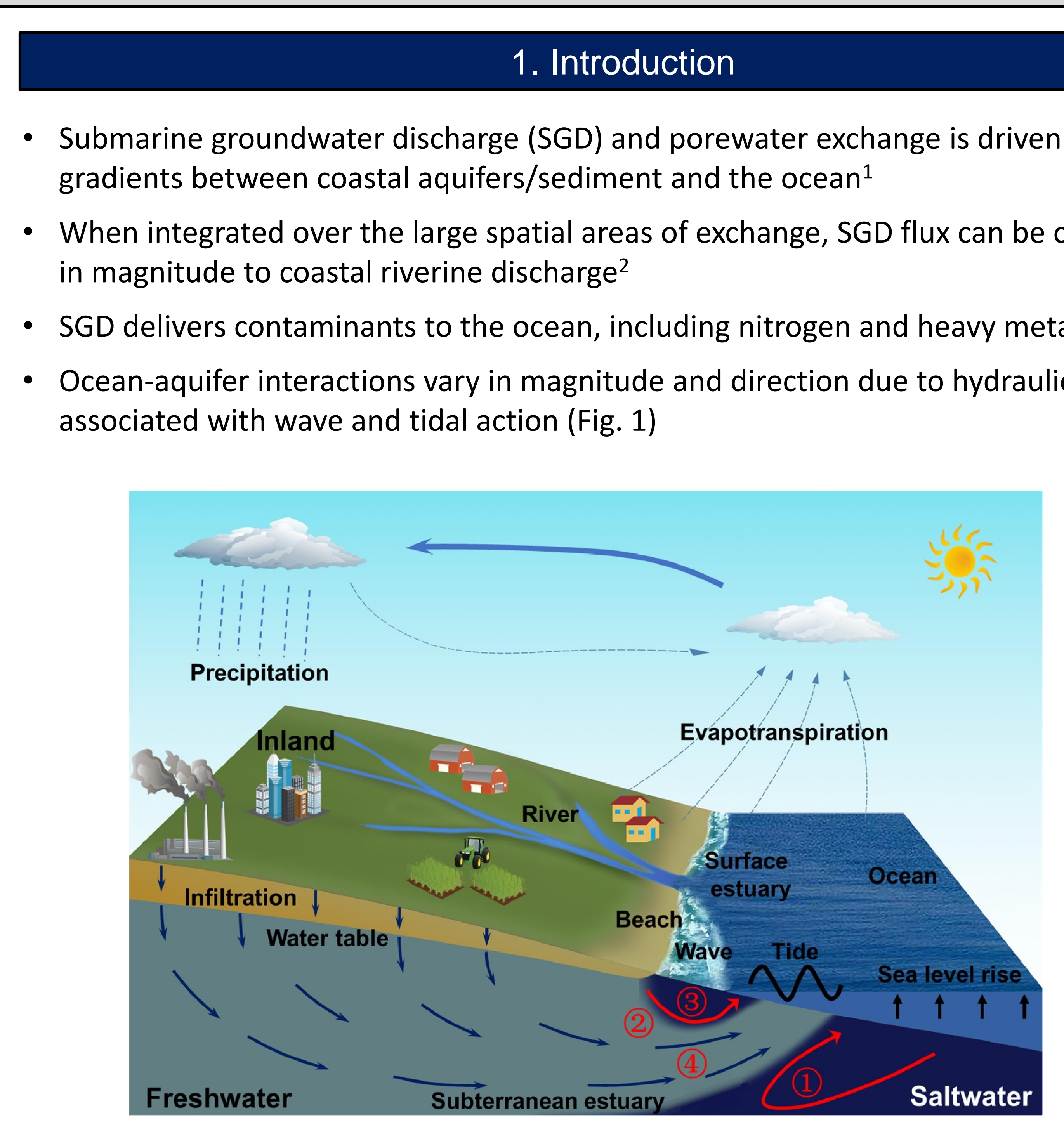

Figure 1: Conceptual model of ocean-aquifer interactions, including: (1) density-driven circulation, (2) tidal
pumping, (3) wave pumping and (4) fresh $S G D$ (modified from Robinson et al.1)

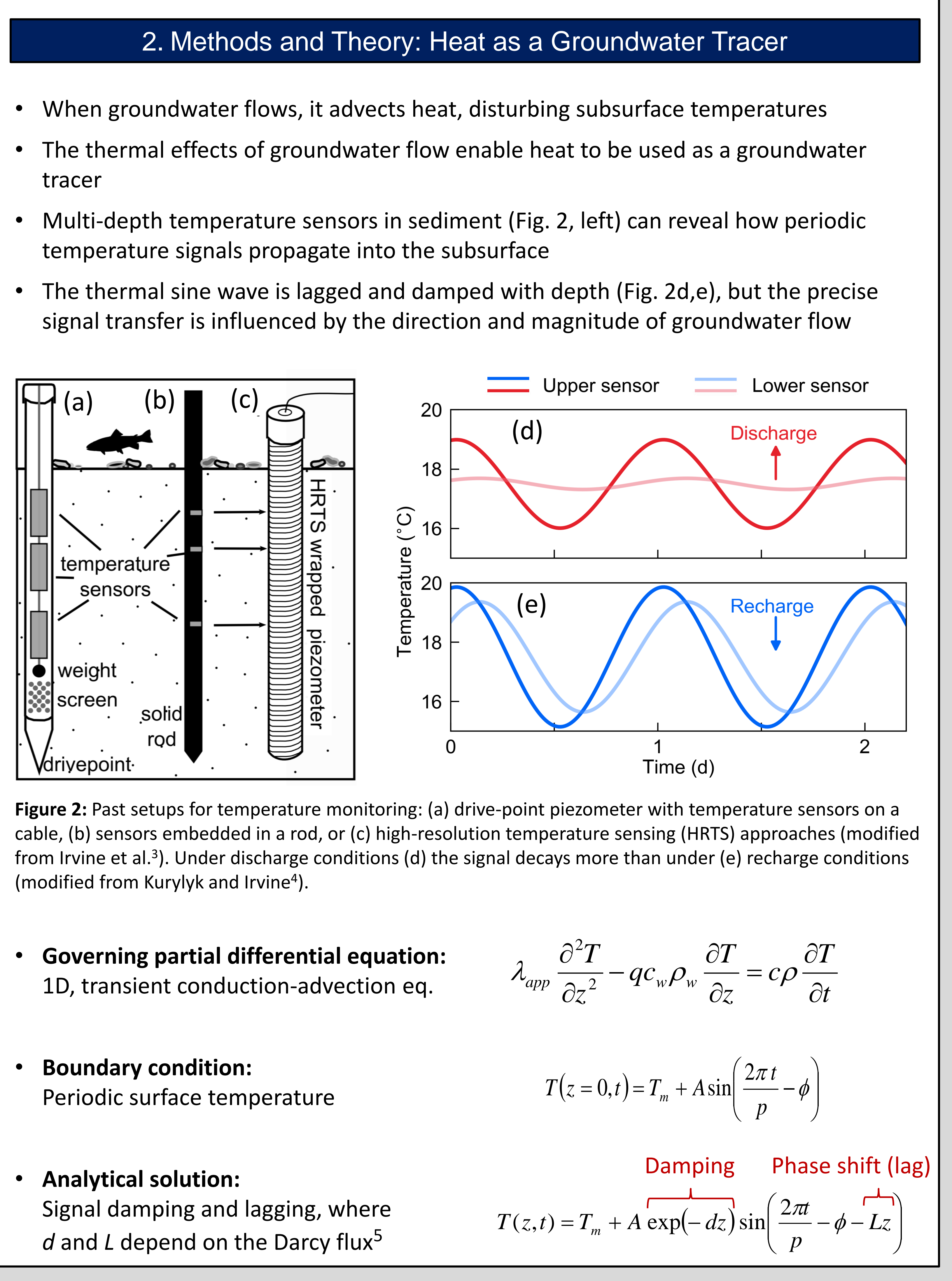

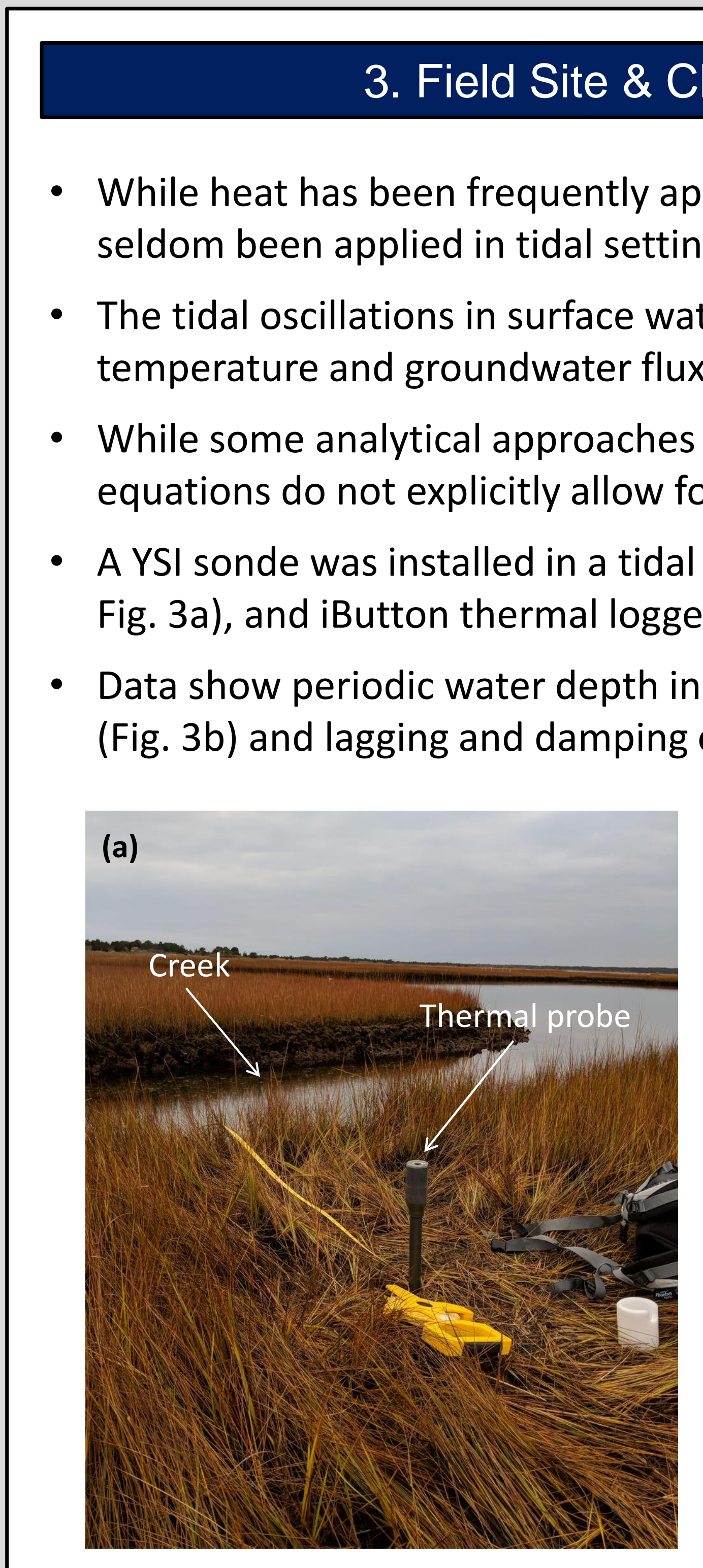

Figure 3: (a) Photograph of the Sage Lot Pond study site with the creek and thermal probe shown. (b) Water

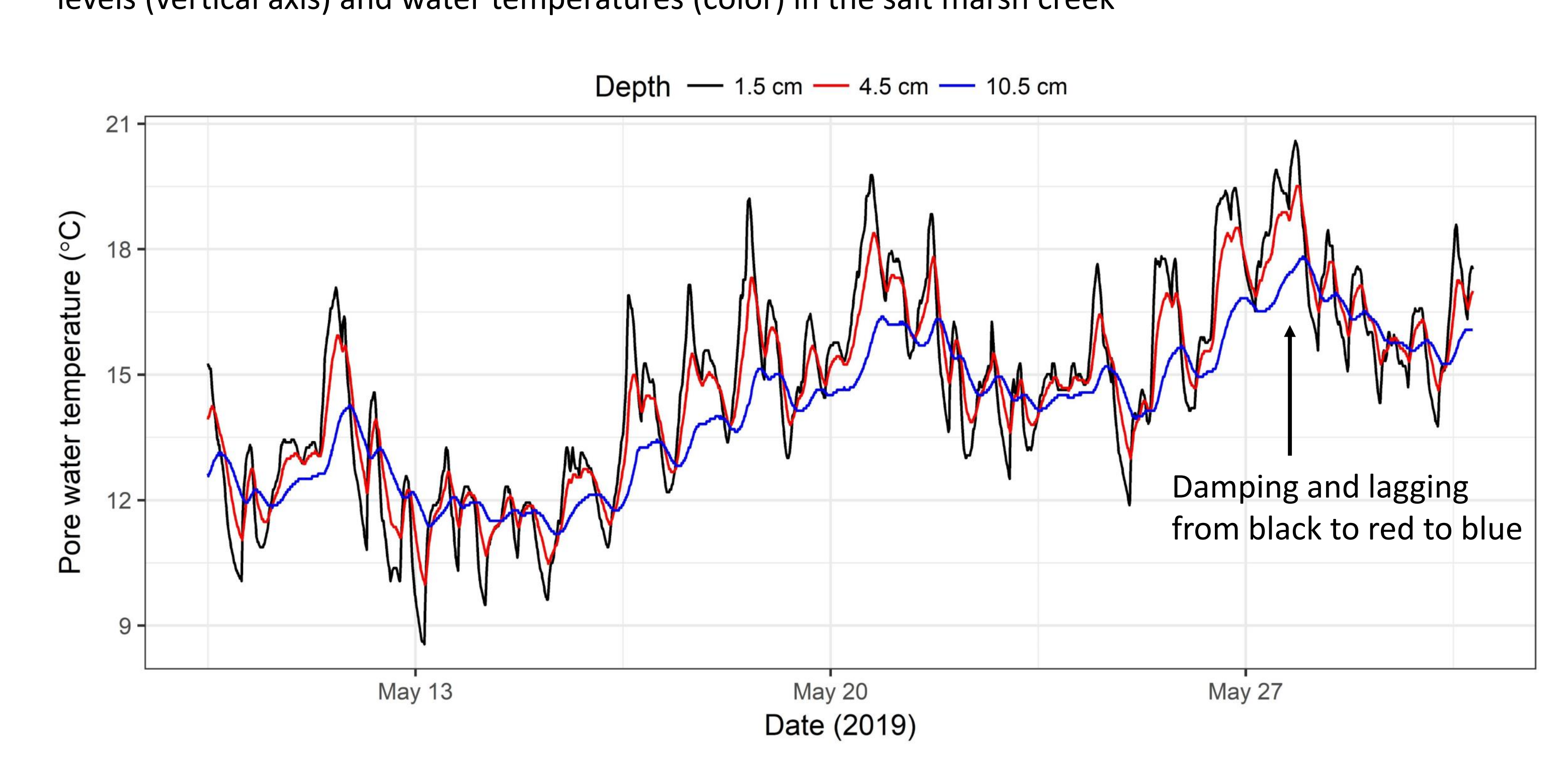

Figure 4: Multi-depth porewater temperatures recorded in Sage Lot Pond in May 2019

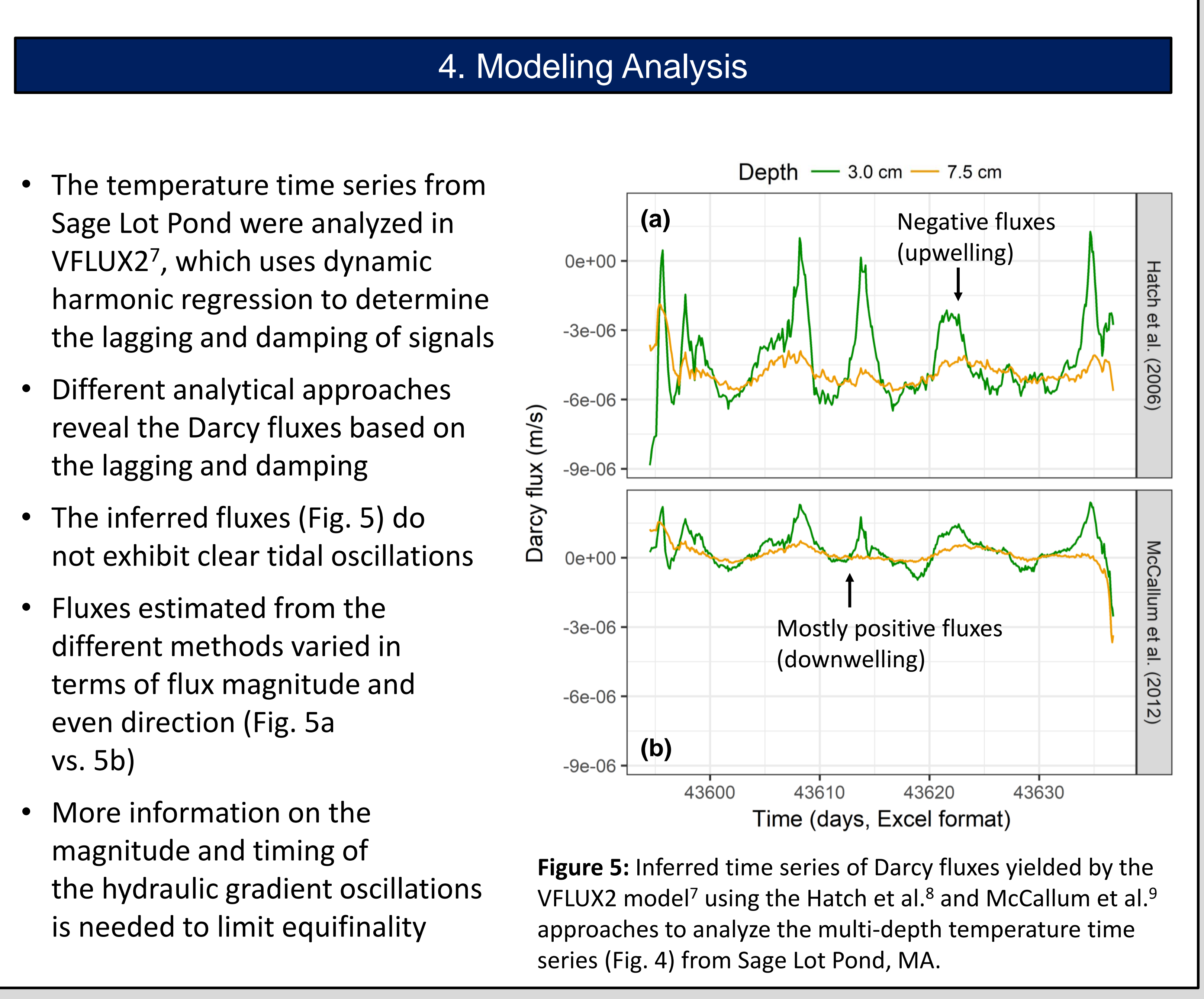

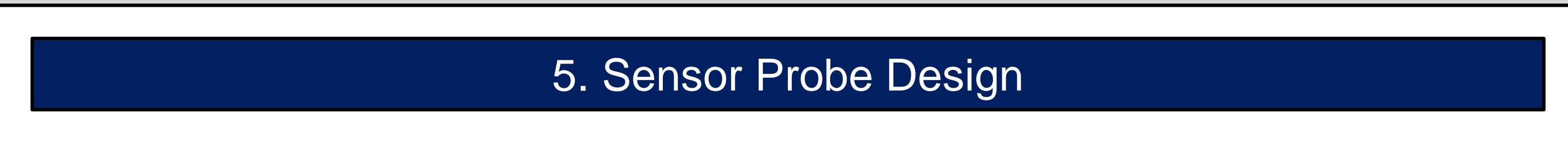

- A novel, inexpensive sensor is proposed that monitors porewater pressure, temperature and conductivity at different depths

Pressure readings reveal the period and magnitude of the tidal fluctuations and thus the hydraulic gradient, while conductivity data indicates whether SGD is fresh or saline Also, the Darcy flux inferred from the thermal data can be used to yield the hydraulic conductivity from the pressure data through Darcy's Law

The design (Fig. 6) incorporates an array of sensors and a programmed microcontroller to record and store the data on a micro SD card

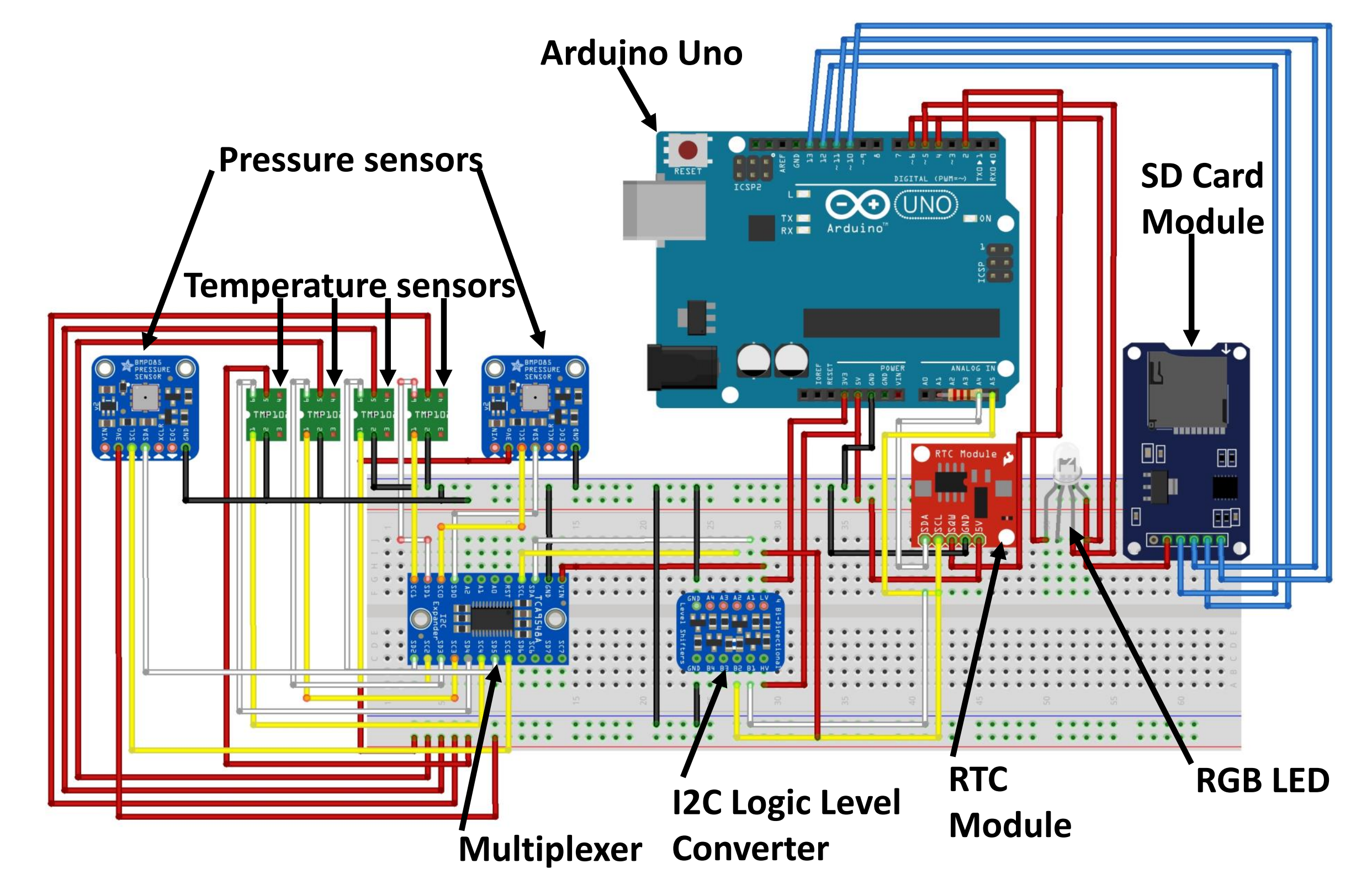

Figure 6: Virtual schembilc and wiring diagram representing the present data logging system with temperature and pressure sensor locations and connetions, Comber in The initial sensor setup is being tested in the laboratory for code performance and sensor operations

Fig. 7 displays the vertical sensor array and probe installation for field deployment

Figure 7: Cartoon of the probe installed in coastal sedimen where water level fluctuates due to tidal influences.
Variables $T, P$, and $C$ represent temperature, pressure at conductivity sensors, respectively, and $z$ represents

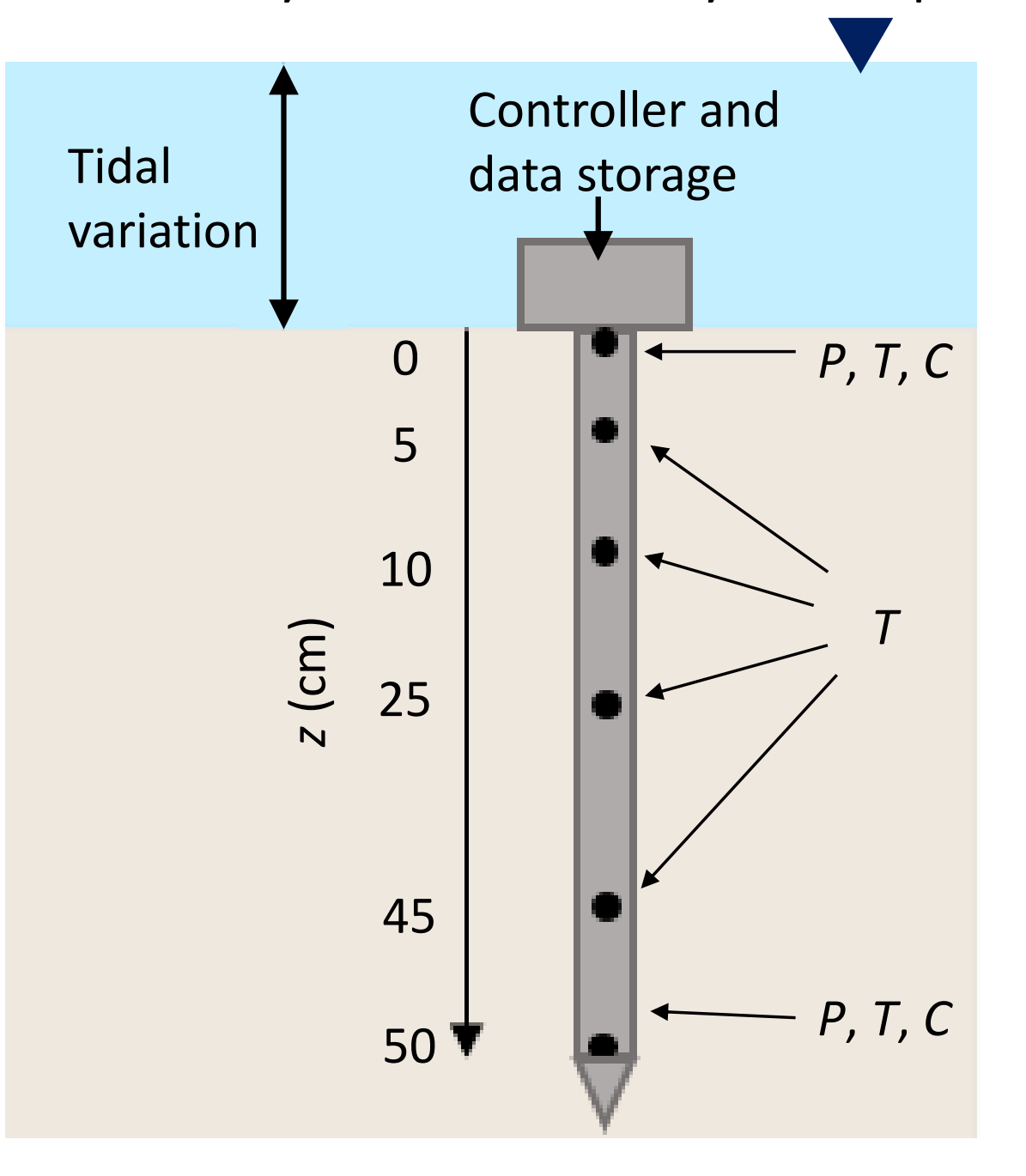

\section{Conclusions and Future Plans}

Groundwater discharge and porewater exchange is important for coastal biogeochemistry and ecosystem health, but its quantification is challenging

- An inexpensive but powerful sensor probe is being developed to reveal the timing and amplitude of groundwater flux response to tidal forcing and determine if SGD is fresh Next steps include finishing the housing unit for the sensors, deploying the probe to collect data in a mega-tidal setting (Bay of Fundy, Nova Scotia), and modifying VFLUX2

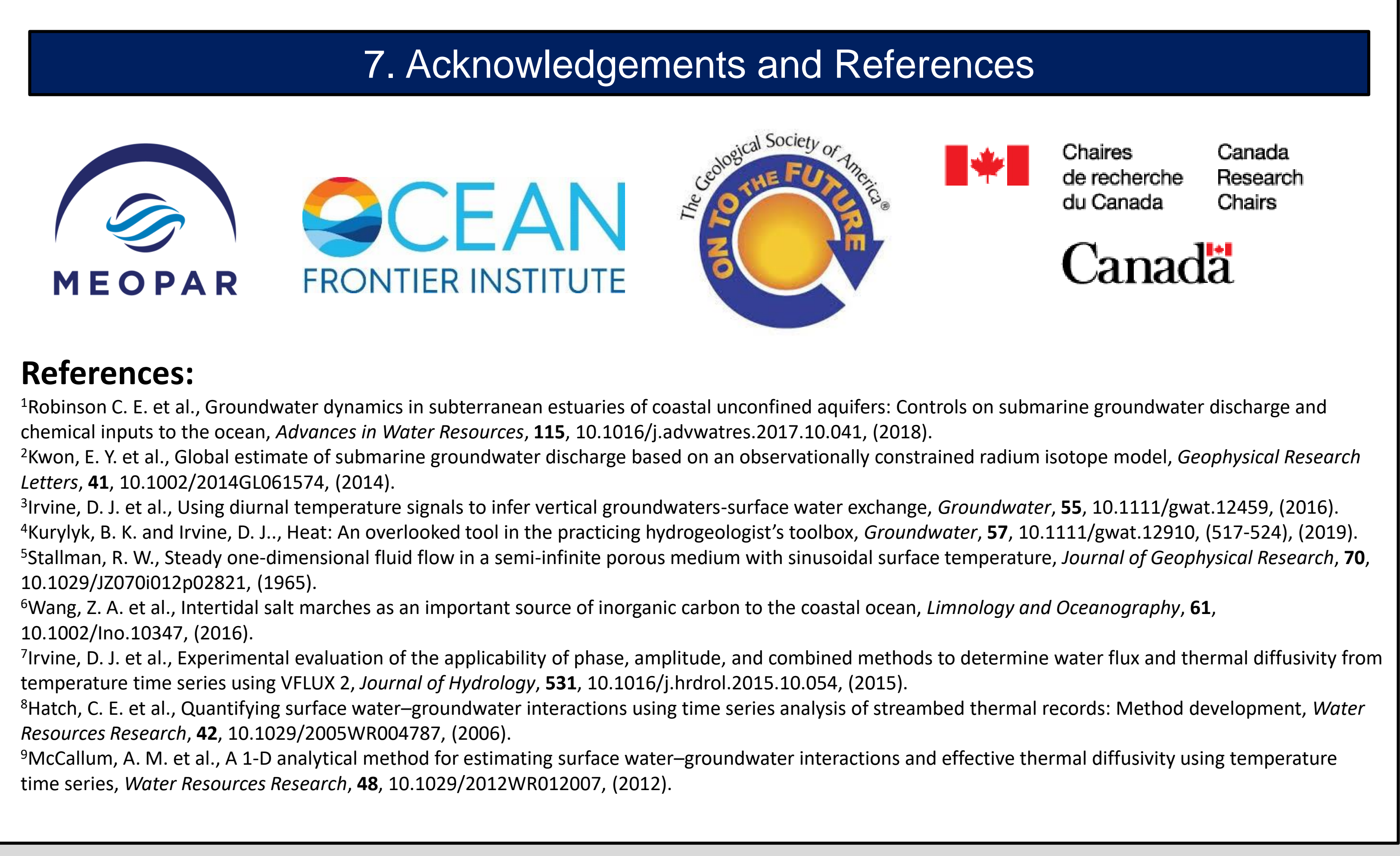

